\title{
1 Passive stiffness of hindlimb muscles in anurans with distinct
}

\section{2 locomotor specializations}

3

$4 \quad$ Nicole Danos*, Emanuel Azizi

5

6 Department of Ecology and Evolutionary Biology, University of California Irvine, 321

7 Steinhaus Hall, Irvine, CA 92625, USA

8

9

10

$11 *$ Corresponding author. Present address: Biology Department, Tufts University, 200

12 Boston Ave., Medford, MA 02155, USA. Tel.: +1 (617) 6270558

13 E-mail address: nicole.danos@tufts.edu (N. Danos).

14

15

16 Manuscript: 20 pages

17 Figures: 6

18 Tables: 3

19 Supplementary data: 1 figure, 1 table

20 


\section{Abstract}

2 Anurans (frogs and toads) have been shown to have relatively compliant skeletal muscles.

3 Using a meta-analysis of published data we have found that muscle stiffness is negatively

4 correlated with joint range of motion when examined across mammalian, anuran and bird

5 species. Given this trend across a broad phylogenetic sample, we examined whether the

6 relationship held true within anurans. We identified four species that differ in preferred

7 locomotor mode and hence joint range of motion (Lithobates catesbeianus, Rhinella marina,

8 Xenopus laevis and Kassina senegalensis) and hypothesized that smaller in vivo angles

9 (more flexed) at the knee and ankle joint would be associated with more compliant

10 extensor muscles. We measured passive muscle tension during cyclical stretching (20\%)

11 around $\mathrm{L}_{0}$ (sarcomere lengths of $2.2 \mu \mathrm{m}$ ) in fiber bundles extracted from cruralis and

12 plantaris muscles. We found no relationship between muscle stiffness and range of motion

13 for either muscle-joint complex. There were no differences in the passive properties of the

14 cruralis muscle among the four species, but the plantaris muscles of the Xenopus and

15 Kassina were significantly stiffer than those of the other two species. Our results suggest

16 that in anurans the stiffness of muscle fibers is a relatively minor contributor to stiffness at

17 the level of joints and that variation in other anatomical properties including muscle-

18 tendon architecture and joint mechanics as well as active control likely contribute more

19 significantly to range of motion during locomotion.

20 Keywords: Locomotion; Jumping; Muscle properties; Joint range of motion 


\section{Introduction}

2 The passive mechanical properties of skeletal muscles can be a significant contributor to

3 force production. These passive properties have been shown to vary substantially among

4 muscles of different species using different modes of locomotion (Azizi and Roberts, 2010).

5 In addition, these passive properties can vary within a single species or individual based on

6 the mechanical function performed by the muscle (Brown et al., 1996a; Azizi, 2014). The

7 passive stiffness of muscles can also change rapidly in response to changes in loading

8 conditions, and stiffness has been shown to increase with strength training, aging and

9 certain pathologies (LaRoche and Connolly, 2006). Despite the significant variation in

10 passive properties, few studies have examined how such changes may impact locomotor

11 capacity at the level of the whole organism.

12 Anuran hindlimbs have been shown to have much more compliant muscles than hindlimbs

13 of other vertebrate taxa (LaRoche and Connolly, 2006; Azizi and Roberts, 2010). The

14 observed compliance is thought to be important for increasing the mechanical work output

15 needed to power the specialized saltatory locomotion in most frogs (Peplowski and Marsh,

16 1997; Azizi and Roberts, 2010). The small plateau of the active force-length curve (Gordon

17 et al., 1966) means that a muscle can only produce maximum force over a narrow $(\sim 15 \%)$

18 range of its length. This suggests that behaviors like jumping where muscles are required

19 to perform large amounts of mechanical work may be limited by the active force-length

20 properties of muscles (Peplowski and Marsh, 1997). Since work is the product of muscle

21 shortening and force, shortening significantly past the optimal length will come at a

22 detriment to force. Therefore it has been suggested that operating at relatively long lengths

23 may be a better strategy for producing higher forces (Azizi and Roberts, 2010). One 
1 challenge in operating at long lengths is that high passive tension likely restricts many

2 muscles from attaining such lengths. However, the relatively compliant muscles of anurans

3 may allow muscles to operate at long length and serve as an important feature of this

4 locomotor system.

5 Similarly, it has been suggested that reduced muscle stiffness and relatively lower passive

6 tension serve to accommodate the very crouched hindlimb posture from which frogs begin

7 their jump (Azizi and Roberts, 2010). In fact, a negative correlation between range of

8 motion and passive muscle stiffness has previously been observed in mammals (Brown et

9 al., 1996a). We performed a meta-analysis of previously published data spanning multiple

10 muscle groups, experimental preparations and phylogenetic groups to examine whether

11 this correlation is a general feature of vertebrate skeletal muscle. We used two distinct

12 measures of muscle stiffness, normalized stiffness and L20, introduced by Azizi and Roberts

13 (2010). Normalized stiffness is the slope of the linear region of a muscle's passive

14 normalized stress-strain curve, where stress is normalized to maximum isometric stress

15 and strain to the muscle's optimum length ( $\left.\mathrm{L}_{0}\right)$. $\mathrm{L}_{20}$ is the muscle length (normalized to $\mathrm{L}_{0}$ )

16 at which passive tension is equal to $20 \%$ of maximum isometric force (Azizi and Roberts,

17 2010). We then used the maximum range of motion reported in the literature for each

18 muscle's main joint of action and plotted our muscle stiffness metrics against these range of

19 motion values (Fig. 1 and Fig. S1 in the supplementary online Appendix). We found a highly

20 significant negative relationship between range of motion and muscle stiffness (Fig. 1). This

21 was true forL20 and normalized stiffness (Fig. $1 ; \mathrm{R}^{2}=0.72$ and 0.54 , respectively; $\mathrm{p}<0.001$ ),

22 indicating that muscles acting at joints with larger ranges of motion could be stretched to

23 longer lengths before developing significant amounts of passive tension and that passive 
1 tension increases less for a given change in length. Our meta-analysis, therefore, suggests

2 the possibility that across a diversity of muscles and phylogenetic groups, muscle

3 properties may passively constrain range of motion around a joint.

4 Given that high joint excursions had been proposed as the functional reason for low muscle

5 compliance in frogs we wanted to test whether the negative correlation between range of

6 motion and muscle stiffness was also true for anurans. We identified four species from four

7 different families, each with a different primary locomotor mode and hence different

8 expected range of motion at the hindlimb joints, and compared the passive muscle stiffness

9 of two extensor muscles (m. cruralis and m. plantaris) to the range of motion of the joints at

10 which they act (knee and ankle, respectively). These species included: (1) the American

11 bullfrog, Lithobates catesbeianus, a jumper; (2) the marine toad, Rhinella marina, a hopper;

12 (3) the African clawed frog, Xenopus laevis, a swimmer; and (4) the Senegal running frog,

13 Kassina senegalensis, a walker/runner. We collected values from the literature describing

14 the in vivo range of motion for the knee and ankle joints of three of these species and

15 collected original data for Kassina frogs during walking using high-speed videography. We

16 then used these range of motion values along with our original measurements of muscle

17 bundle stiffness to test the hypothesis suggested by our meta-analysis (Fig. 1): a larger

18 range of motion at a joint will correlate with a more compliant extensor muscle acting at

19 that joint. In addition, we quantified differences in muscle architecture and muscle moment

20 arms to examine other potential determinants of passive joint mechanics. Testing these

21 predictions will allow us to better understand the relationship between the motion of joints

22 during locomotion and the underlying passive properties of muscles. 


\section{2. Materials and methods}

2 Animals were maintained at UC Irvine according to IACUC-approved protocols. Following

3 euthanasia by MS-222 overdose and double pithing, the animals were first tested for joint

4 stiffness and muscle moment arm calculations. Fresh tissue samples were then dissected

5 out for muscle fiber bundle stiffness tests. Cruralis and plantaris muscles were also

6 dissected from animals euthanized for other experiments to measure muscle mass,

7 proportion of total extensor mass and pennation angle. The body mass (mean \pm sem) of the

8 Lithobates frogs was $129.5 \pm 2.1 \mathrm{~g}(\mathrm{n}=7)$, Rhinella $104.5 \pm 8.1 \mathrm{~g}(\mathrm{n}=7)$, Kassina $1.95 \pm 0.22$

$9 \mathrm{~g}(\mathrm{n}=10)$ and Xenopus $92.8 \pm 7.8 \mathrm{~g}(\mathrm{n}=8)$. Xenopus frogs originated from breeder facilities

10 while the other three species were all wild caught and purchased from herpetological

11 vendors.

\section{2.1. Joint range of motion}

14 Range of motion information for the knee and ankle joints was obtained from the literature

15 for L. pipiens as a representative ranid species while swimming (Richards, 2010) and

16 jumping (Chadwell et al., 2002), for X. laevis during swimming (Richards, 2010) and for $R$.

17 marina during hopping (Chadwell et al., 2002) and swimming (Richards, 2010). Joint

18 angles for the knee and ankle of K. senegalensis were obtained from high-speed video

19 collected in our laboratory as part of another study. Briefly, animals were allowed to move

20 freely in a filming arena (approximately $30 \mathrm{~cm}$ long and $15 \mathrm{~cm}$ wide) and filmed dorsally

21 and laterally with Phantom M120 cameras (Vision Research Inc., Wayne, NJ, USA) at 200

22 frames/second. Joint angles during terrestrial locomotion were determined three-

23 dimensionally (Chadwell et al., 2002; present study), while swimming joint angles 
1 (Xenopus) were obtained from dorsal images only. The maximum range of motion during a

2 single locomotor mode was used as the predictor of muscle stiffness.

$4 \quad$ 2.2. Joint stiffness

5 Animals were euthanized and the skin on their legs removed. The animals were placed on a

6 platform and the limb segment distal to the joint whose stiffness we were characterizing

7 was attached to the ergometer (model 305C-LR; Aurora Scientific Inc., Ontario, Canada).

8 The limb and the ergometer were aligned such that the motion of the joint and the lever of

9 the ergometer were in the same plane. The distance between the joint and its attachment

10 point to the ergometer lever, normalized to the limb segment length, was statistically

11 indistinguishable among the four species. The joint was moved through its full range, which

12 varied among the investigated species due to different leg morphologies, in approximately

$1315^{\circ}$ increments. The static force at each joint angle increment was recorded by the

14 ergometer and a dorsal photograph was taken to quantify joint angle. Ergometer data were

15 collected at $1000 \mathrm{~Hz}$ using a 16 bit data acquisition board (National Instruments, Austin,

16 TX, USA) and IGOR-Pro software (Wavemetrics, Portland, OR, USA).

17

18 2.3. Passive tension in muscle bundles

19 Muscle fiber bundles were dissected out of the muscle of interest using iris spring scissors

20 and fine forceps and mounted at their two ends using micro vessel clamps. One clamp was

21 attached to a three-axis micromanipulator while the other was attached to an ergometer

22 (model 300C-LR; Aurora Scientific Inc.) that controlled the length of the fiber bundle and

23 measured its tension. Data were collected at $1000 \mathrm{~Hz}$ using a 16 bit National Instruments 
1 data acquisition board and IGOR-Pro software. A $650 \mathrm{~nm}$ laser (Thorlabs, Newton, NJ, USA)

2 was shone at the muscle and the diffraction pattern created by the sarcomere structure

3 was captured on a calibrated screen, the position of which was adjustable but generally

4 positioned $\sim 2 \mathrm{~cm}$ away from the muscle. The laser diffraction pattern created by the

5 muscle bundle at different lengths and hence ergometer positions was captured using a

6 digital camera. From these images we calculated the sarcomere length in $\mu \mathrm{m}$ from the

7 distance between the bands of the diffraction pattern according to equation (1) (Lieber et

8 al., 1992),

$9 S L=n \lambda /\left(\sin \left(\tan ^{-1}\left(x_{n} / h\right)\right)\right)$,

10 where $S L$ is sarcomere length, $n$ is the number of bands away from the central band, $\lambda$ is the

11 laser wavelength, $x_{n}$ the distance between the central and $n^{\text {th }}$ band and $\mathrm{h}$ the distance of the

12 diffraction pattern (screen) from the muscle bundle.

13 Force on the bundle was normalized by its cross-sectional area, which was calculated using

14 the bundle's mass, density of $1.06 \mathrm{~g} / \mathrm{cm}^{3}$ (Mendez and Keys, 1960) and bundle length at

15 sarcomere length of $2.2 \mu \mathrm{m}$. The stress-sarcomere length relationship was determined for

16 the third stretching cycle from each muscle fiber bundle examined and a curve was fitted to

17 it according to Brown et al. (1996b) using equation (2):

18 Stress $(L)=c k \ln ((\exp (L-L r) / k)+1)$, Eq. (2)

19 Where $L$ is the sarcomere length, and $c, k$ and $L_{r}$ are constants that describe the shape of the

20 stress-strain curve. Muscles with higher stiffness will have a higher slope in the linear

21 region of the stress-strain curve $(c)$, a smaller radius of curvature in the exponential part of

22 the stress-strain curve $(k)$ and a lower length at which tension begins to develop $\left(L_{r}\right)$.The

23 three fit parameters that described the stress-sarcomere length relationship were 
1 recorded for each bundle, and a one-way ANOVA was performed on the pool of individual

2 fit values. The ANOVA was followed by a Tukey HSD test if a significant effect of species on

3 the fit value was found.

\section{2.4. Muscle-tendon architecture}

6 In order to account for other determinants that could passively limit joint angles we further

7 quantified muscle-tendon architecture for the cruralis and plantaris. The passive torque

8 exerted on a joint is the product of the passive force in the muscle and the moment arm of

9 that muscle around the joint. To measure muscle moment arms, animals were euthanized

10 and the upper body firmly fixed to a rubber-lined dissection tray. The skin was then

11 removed from the entire leg. The tissues directly connected to the cruralis (fascia lata,

12 tensor fasciae latae and gluteus maximus) were cut in cross-section but left in place. The

13 cruralis originates on the ilium just ventral to the acetabulum and inserts via a broad

14 aponeurosis that covers the knee onto the tibiofibular (Prikryl et al., 2009), so to account

15 for the small moment arm around the hip, the moment arm of the cruralis muscle at the

16 knee was determined with the hip joint kept constant at $90^{\circ}$. Moment arms were measured

17 for 6 cruralis and 4 plantaris muscles for Kassina, 5 cruralis and 7 plantaris for Lithobates, 4

18 cruralis and 7 plantaris for Rhinella and 3 cruralis and 8 plantaris for Xenopus. Moment

19 armswere calculated as muscle length change (normalized by bone length, femur for the

20 cruralis and tibiofibula for the plantaris) per unit change in joint angle. This was done by

21 placing two markers superficially along the direction of muscle fascicles and positioning

22 the limb with the hip at $90^{\circ}$ and the knee fully flexed. Photographs were then taken at small

23 increments of increased knee extension. For the plantaris, a similar protocol was followed 
1 but in this case no other muscles needed to be dissected, the knee joint was always at

$290^{\circ}$ and the ankle was moved through its range of motion from full flexion to full extension.

3 The change in muscle length with increasing joint angle was obtained from the middle

4 region of the joint range of motion when connective tissue was not likely to be loaded

5 under tension (Lieber and Brown, 1992).

6 Muscle mass was recorded as a proportion of total extensor muscle group mass. Cross-

7 sectional area, which would determine the total amount of passive force acting on a joint,

8 was calculated using the mass, length and density of the muscle (Mendez and Keys, 1960).

9 To measure pennation angle, muscles were fixed at their resting length (with joints at $90^{\circ}$ )

10 in $10 \%$ formalin. The fixed muscle was bisected sagittally, from one aponeurosis to the

11 other, and the angle of the fiber relative to the aponeurosis was measured at several points

12 in the muscle using ImageJ (Rasband, 1997-2014).

\section{3. Results}

\section{3.1. Joint range of motion}

16 We calculated the range of motion at a joint as the difference between the maximum and

17 minimum joint angle. When multiple modes were reported such as in the case of Lithobates

18 we used the values that yielded the highest range of motion. We used the following

19 minimum and maximum joint angles to quantify the range of motion at the knee (Table 1):

$2026^{\circ}$ to $153.7^{\circ}$ for Lithobates (Chadwell et al., 2002; Richards, 2010), $40.9^{\circ}$ to $133^{\circ}$ for

21 Rhinella (Chadwell et al., 2002; Richards, 2010), $78.9^{\circ}$ to $130.9^{\circ}$ for Xenopus (Richards,

222010 ) and $47.5^{\circ}$ to $123.7^{\circ}$ for Kassina. The following minimum and maximum joint angles

23 describe the ankle (Table 1): $52^{\circ}$ to $168.5^{\circ}$ for Lithobates (Chadwell et al., 2002; Richards, 
12010 ), $55^{\circ}$ to $168^{\circ}$ for Rhinella (Chadwell et al., 2002; Richards, 2010 ), $54.2^{\circ}$ to $179.8^{\circ}$ for

2 Xenopus (Richards, 2010) and $19.9^{\circ}$ to $94.5^{\circ}$ for Kassina.

3

$4 \quad 3.2$. Joint passive stiffness

5 Joint stiffness was best described by a quadratic relationship between force and angle.

6 Passive force was high at low and high angles (joint flexion and extension, respectively)

7 with a region of low force at intermediate angles (Fig. 2). Joint stiffness was assessed using

8 two criteria: the angle at which joint force was at a minimum and the quadratic coefficient

9 of the curve of best fit, which describes the width and slope of the force-angle relationship.

10 At the knee, all four species differed significantly in the minimum force angle with Xenopus

11 having the smallest angle and Lithobates the highest (Table 1). The exponent of the curve

12 that described the stiffness of the ankle joint also had a significant species effect $(\mathrm{P}=$

13 0.0078). A Tukey HSD test showed that Lithobates had significantly higher quadratic

14 coefficients, indicating a narrower curve with a steeper slope (Table1 and Fig. 2).

15 The ankle joint had force versus angle curves with minima that differed significantly among

16 all four species. For the ankle, Rhinella had a curve with a minimum at the lowest angle

17 while Xenopus at the largest joint angle (Table 1). The fits to the data from Kassina and

18 Xenopus had the largest coefficients indicating the narrowest and steepest curve. Lithobates

19 had the most compliant ankle as measured by the coefficient, with Rhinella having an

20 intermediate value (Table 1).

21 Overall, Kassina and Lithobates had the stiffest knee joints while Xenopus had the most

22 compliant knee. However, Xenopus along with Kassina had the stiffest ankle joints while 
1 Lithobates had the most compliant ankle. The observed patterns of joint stiffness did not

2 correlate with the maximum range of motion used during locomotion in the four species.

3

$4 \quad$ 3.3. Muscle bundle passive stiffness

5 The stiffness of the cruralis muscle, as measured at the fiber bundle level, did not vary

6 among the four species examined here (Table 2 and Fig. 4). The plantaris muscle, however,

7 showed significant differences among species in the fit parameters $\mathrm{k}$ and $\operatorname{Lr}$ (ANOVA, $\mathrm{p}=$

80.03 and 0.0002 , respectively). Rhinella had a significantly higher $k$ value (Table 2 and Fig.

9 4), indicating a higher radius of curvature at the exponential part of the curve, while

10 Xenopus had the smallest $k$ coefficient indicating a muscle that developed passive force at a

11 faster rate (Brown et al., 1996b). Rhinella also had a longer $L_{r}$ than the other species (Tukey

12 HSD, $\mathrm{p}<0.003$ for all comparisons) indicating a muscle that could be stretched to longer

13 lengths before developing appreciable tension. The slope of the curve at longer lengths, $c$,

14 did not vary significantly among all the species (Table 2).

15 There was no significant relationship between range of motion and muscle bundle stiffness.

17 3.4. Muscle architecture

18 Muscle cross-sectional area of the cruralis, calculated from measurements of muscle mass

19 and length and corrected for body mass, was significantly higher for the Kassina compared

20 to all the other species (ANOVA, $\mathrm{P}=4.03 \times 10^{-8}$; Table 3 ). Body mass corrected cross-

21 sectional area of the plantaris was similar for Xenopus and Kassina, but significantly distinct

22 from that of Rhinella and Lithobates (ANOVA, $\mathrm{P}=3.42 \times 10^{-7}$; Table 3). 
1 Pennation angle did not differ significantly among species for either the plantaris or

2 cruralis $\left(19.7 \pm 1.8^{\circ}\right.$ and $23.1 \pm 1.9^{\circ}$, respectively $)$. However, the magnitude of distal

3 moment arms of both muscles, normalized by femur or tibiofibula length, showed a

4 significant species effect (ANOVA, $P=0.0002$ ). The moment arms of the cruralis fell into

5 two groups: Rhinella and Xenopus had relatively longer moment arms around the knee (4.8

$6 \times 10^{-4}$ and $3.9 \times 10^{-4}$ femur lengths $/^{\circ}$, respectively) than Kassina and Lithobates (9.8 x 10-5

7 femur lengths $/{ }^{\circ}$ for both species; Fig. $5 \mathrm{~A}$ ). The moment arms of the plantaris around the

8 ankle had a marginally significant effect of species (ANOVA, $P=0.087$ ), driven by a

9 significantly shorter moment arm in the Kassina $\left(4.8 \times 10^{-5}\right.$ tibiofibula lengths $\left./{ }^{\circ}\right)$ compared

10 to the other three species $\left(4.3 \times 10^{-4}, 4.0 \times 10^{-4}\right.$ and $2.8 \times 10^{-4}$ tibiofibula lengths $/{ }^{\circ}$ for

11 Rhinella, Lithobates and Xenopus, respectively; Fig. 5B). The mean ( \pm sem) femur lengths

12 used to normalize absolute moment arm measurements were as follows: $39.6 \pm 1.7 \mathrm{~mm}$ for

13 Rhinella, $48.2 \pm 0.3 \mathrm{~mm}$ for Lithobates, $41.7 \pm 1.2 \mathrm{~mm}$ for Xenopus and $11.3 \pm 0.5 \mathrm{~mm}$ for

14 Kassina. The mean ( \pm sem) tibiofibula lengths used to normalize absolute moment arm

15 measurements were as follows: $41.2 \pm 1.0 \mathrm{~mm}$ for Rhinella, $45.4 \pm 0.7 \mathrm{~mm}$ for Lithobates,

$1634.2 \pm 0.6 \mathrm{~mm}$ for Xenopus and $10.3 \pm 0.7 \mathrm{~mm}$ for Kassina.

\section{4. Discussion}

19 We tested whether joint range of motion explained differences in muscle stiffness within a

20 highly specialized group of animals, the Anura, in a manner similar to what our meta-

21 analysis of large phylogenetic groups revealed (Fig. 1) and to what was shown for muscles

22 from a single mammalian species (Brown et al, 1996a). Based on the significant correlation

23 between joint range of motion and muscle stiffness metrics from our meta-analysis (Fig. 1) 
1 we had hypothesized that species with higher range of motion would have lower

2 normalized stiffness and develop $20 \%$ of $\mathrm{P}_{0}$ passively at longer lengths. Although we found

3 significant variation in muscle stiffness among species, we found no significant relationship

4 between range of motion and muscle stiffness metrics within anurans (Fig. 6). We found

5 that species also differed in the stiffness of their ankle and knee joints, although again there

6 was no clear relationship between joint stiffness and range of motion at the knee (Table 1).

7 Together these results suggest that mechanisms other than muscle stiffness may constrain

8 joint mechanics in anurans.

9 Our measurements of passive muscle stiffness are consistent with previously published

10 results (Brown et al., 1996a, b; Azizi and Roberts, 2010) (Fig. 6); anuran skeletal muscles

11 are highly compliant when compared to mammals and birds. It is therefore not entirely

12 surprising that passive muscle properties in frogs are largely decoupled from passive

13 torques measured at the level of joints. Extramuscular connective tissues such as

14 aponeuroses, fascias, tendons and ligaments can increase overall stiffness (Johns and

15 Wright, 1962; Gajdosik, 2001; Kawakami et al., 2008; Maas and Sandercock, 2010).

16 Similarly, joint facet morphology has been shown to contribute significantly to joint

17 stiffness of the vertebral column and is therefore a likely contributor to the ankle and knee

18 stiffness of the species we examined (Koob and Long, 2000; Nowroozi and Brainerd, 2013;

19 Molnar et al., 2014). Our results suggest that passive elements at higher levels of

20 organization likely determine the passive properties of the joints.

21 Among the terrestrial frogs (excluding X. laevis), the quadrupedal walking/running frog

22 Kassina displayed some specializations that are likely related to its unique mode of

23 locomotion. Kassina had the smallest range of motion at both the knee and the ankle and 
1 also had some of the stiffest joints (Table 1). The natural range of motion for this species

2 overlaps with the steepest regions of the joint stiffness curve (Figs. 2 and 3), indicating that

3 during normal locomotion Kassina operates in the region of high knee and ankle stiffness.

4 During our filming of this species we observed that when moving quadrupedally, the

5 hindlimbs remain highly flexed while most of the foot movement is due to excursion at the

6 tarso-metatarsal joint. Having passively stiff joints may therefore reduce the active postural

7 work that ankle flexors need to perform, especially at high speeds (Ahn et al., 2004).

8 We found different patterns of muscle stiffness for the knee and ankle extensors we

9 examined. Although we found no significant difference between the passive properties of

10 the knee extensor (cruralis), there were significant species differences in the passive

11 properties of the ankle extensor (plantaris). This may be a result of the fact that the

12 plantaris is the main ankle flexor in all species (Dunlap, 1960; Kamel et al., 1996), while

13 there are at least two other muscles, tensor fasciae latae and gluteus magnus, that act as

14 synergists to the cruralis (Gillis and Biewener, 2000). In the species we examined, the

15 plantaris constituted more than $90 \%$ of the total ankle extensor mass while the cruralis

16 contributed only about $65 \%$ of the knee extensors (Table 3). Most anurans routinely swim

17 in addition to hopping, jumping or walking (Zug, 1978; Marsh, 1994; Gillis and Biewener,

18 2000; Richards, 2010), and the role of each muscle might differ with locomotor mode, for

19 example, being more postural in function during walking than swimming (Gillis and

20 Biewener, 2000; Chadwell et al., 2002). The multiple functional requirements along with

21 the lack of stiffness variation among species suggest that this muscle maintains a generalist

22 functional morphology. 
1 Xenopus, a specialized swimmer and the only species that never uses saltatory locomotion,

2 had the stiffest plantaris muscle. Xenopus is a fully aquatic frog with the highest range of

3 motion at the ankle. We measured L20 and normalized stiffness values for the Xenopus

4 plantaris that resembled those of small mammals (Figs. 1 and 6). During swimming, ankle

5 extension contributes significantly more than knee extension to thrust production

6 (Richards, 2010). In Xenopus, in particular, swimming propulsion is completely driven by

7 foot rotation (Richards, 2010), in contrast to semiaquatic species where foot translation is

8 a more significant contributor to propulsion. While foot translation is the product of

9 extension at many joints (Richards, 2010), foot rotation is solely due to the shortening

10 action of the plantaris muscle. Higher muscle stiffness may therefore be advantageous for

11 resisting the hydrodynamic load on the foot as it rotates through the water (Richards,

12 2011) while keeping the muscle at an optimal length for high force production.

13 Our study demonstrates that the relatively compliant muscles of anurans do not constrain

14 joint range of motion during locomotion. Therefore, our meta-analysis trend appears to be

15 driven by specializations between major animal groups whose locomotor specializations

16 are characterized by extremes in both range of motion and muscle passive properties. At

17 the one extreme are the cursorial horses with their high muscle stiffness and low range of

18 motion while saltatory anurans have evolved extremely compliant muscles and high range

19 of motion. The functional advantages of extremely high or low muscle stiffness are

20 therefore not related to the passive control of joints but are likely related to the dominant

21 function of muscle during specialized types of locomotion (Azizi, 2014). While high muscle

22 stiffness may contribute to overall increased limb stiffness in horses, potentially

23 contributing to the elastic energy usage (McGuigan and Wilson, 2003), low muscle stiffness 
1 in frogs may instead be linked to the increased power requirements for jumping (Azizi and

2 Roberts, 2010).

3

\section{Acknowledgements}

5 We would like to thank two undergraduate students, Christina Cheung and Michael

6 Kennedy, for their valuable help during these experiments. The constructive comments of

7 two anonymous reviewers greatly improved our manuscript. This research was funded by

$8 \quad$ NSF grant1051691.

9

10 Appendix A. Supplementary data

11 Supplementary data associated with this article can be found in the online version at doi:

12 \#\#\#.

13

14 


\section{References}

Ahn, A.N., Furrow, E., Biewener, A.A., 2004. Walking and running in the red-legged running frog, Kassina maculata. J. Exp. Biol. 207, 399-410.

Azizi, E., 2014. Locomotor function shapes the passive mechanical properties and operating lengths of muscle. Proc. R. Soc. B 281, 20132914. http://dx.doi.org/10.1098/rspb.2013.2914

Azizi, E., Roberts, T.J., 2010. Muscle performance during frog jumping: influence of elasticity on muscle operating lengths. Proc. R. Soc. B 277, 1523-1530.

Brown, I.E., Liinamaa, T.L., Loeb, G.E., 1996a. Relationships between range of motion, lo, and passive force in five strap-like muscles of the feline hind limb. J. Morphol. 230, 6977.

Brown, I.E., Scott, S.H., Loeb, G.E., 1996b. Mechanics of feline soleus: II. Design and validation of a mathematical model. J. Muscle Res. Cell. Motil. 17, 221-233.

Chadwell, B.A., Hartwell, H.J., Peters, S.E., 2002. Comparison of isometric contractile properties in hindlimb extensor muscles of the frogs Rana pipiens and Bufo marinus: functional correlations with differences in hopping performance. J. Morphol. 251, 309322.

Dunlap, D.G., 1960. The comparative myology of the pelvic appendage in the Salientia.J. Morphol. 76, 1-76.

Gajdosik, R.L., 2001. Passive extensibility of skeletal muscle: review of the literature with clinical implications. Clin. Biomech. 16, 87-101.

Gillis, G.B.,Biewener, A.A., 2000. Hindlimb extensor muscle function during jumping and swimming in the toad (Bufo marinus). J. Exp. Biol. 203, 3547-3563.

Gordon, A.M., Huxley, A.F., Julian, F.J., 1966. The variation in isometric tension with sarcomere length in vertebrate muscle fibres. J. Physiol. 184, 170-192.

Johns, R.J., Wright, V., 1962. Relative importance of various tissues in joint stiffness. J. Appl. Physiol. 17, 824-828.

Kamel, L.T., Peters, S.E., Bashor, D.P., 1996. Hopping and swimming in the leopard frog, Rana pipiens: II. A comparison of muscle activities. J. Morphol. 230, 17-31.

Kawakami, Y., Kanehisa, H.,Fukunaga, T., 2008. The relationship between passive ankle plantar flexion joint torque and gastrocnemius muscle and achilles tendon stiffness: implications for flexibility. J. Orthop. Sports Phys. Ther. 38, 269-276.

Koob, T.J., Long, J.H., 2000. The vertebrate body axis: evolution and mechanical function. Am. Zool. 40, 1-18.

LaRoche, D.P., Connolly, D.A.J., 2006. Effects of stretching on passive muscle tension and response to eccentric exercise. Am. J. Sports Med. 34, 1000-1007.

Lieber, R.L., Brown, C.G., 1992. Sarcomere length-joint angle relationships of seven frog hindlimb muscles. Acta Anat. (Basel) 145, 289-295.

Maas, H., Sandercock, T.G., 2010. Force transmission between synergistic skeletal muscles through connective tissue linkages. J. Biomed. Biotech. 2010, 575672. http://dx.doi.org/10.1155/2010/575672.

Marsh, R.L., 1994. Jumping ability of anuran amphibians. Adv. Vet. Sci. Comp. Med. 38B, 51111. 
McGuigan, M.P., Wilson, A.M., 2003. The effect of gait and digital flexor muscle activation on limb compliance in the forelimb of the horse Equus caballus. J. Exp. Biol. 206, 13251336.

Mendez, J., Keys, A., 1960. Density and composition of mammalian muscle. Metabolism 9, 184-188.

Molnar, J.L., Pierce, S.E., Hutchinson, J.R., 2014. An experimental and morphometric test of the relationship between vertebral morphology and joint stiffness in Nile crocodiles (Crocodylus niloticus). J. Exp. Biol. 217, 758-768.

Nowroozi, B.N., Brainerd, E.L., 2013. X-ray motion analysis of the vertebral column during the startle response in striped bass, Morone saxatilis. J. Exp. Biol. 216, 2833-2842.

Peplowski, M.M., Marsh, R.L., 1997. Work and power output in the hindlimb muscles of Cuban tree frogs Osteopilus septentrionalis during jumping. J. Exp. Biol. 200, 28612870.

Prikryl, T., Aerts, P., Havelková, P., Herrel, A., Rocek, Z., 2009.Pelvic and thigh musculature in frogs (Anura) and origin of anuran jumping locomotion. J. Anat. 214, 100-139.

Rasband, W.S., 1997-2014. ImageJ.U.S. National Institutes of Health, Bethesda, Maryland, USA, http://imagej.nih.gov/ij/.

Richards, C.T., 2010. Kinematics and hydrodynamics analysis of swimming anurans reveals striking inter-specific differences in the mechanism for producing thrust. J. Exp. Biol. 213, 621-634.

Richards, C.T., 2011. Building a robotic link between muscle dynamics and hydrodynamics. J. Exp. Biol. 214, 2381-2389.

Richards, C.T., Biewener, A.A., 2007. Modulation of in vivo muscle power output during swimming in the African clawed frog (Xenopus laevis). J. Exp. Biol., 210, 3147-3159.

Zug, G.R., 1978. Anuran locomotion - structure and function. 2. Jumping performance of semiaquatic, terrestrial, and arboreal frogs. Smithson. Contrib. Zool., 276, 1-31. 
Fig. 1. Meta-analysis of mammalian, anuran and bird hindlimb muscle stiffness as a function of joint range of motion. (A) $\mathrm{L}_{20}$ (the length at which the muscle develops $20 \%$ of $\mathrm{P}_{0}$ passively, normalized to $\left.\mathrm{L}_{0}\right)$, becomes longer with increased range of motion $(\mathrm{p}<0.001)$. (B) Normalized stiffness (Young's modulus normalized to $\mathrm{P}_{0}$ and $\mathrm{L}_{0}$ ) decreases with increasing range of motion $(\mathrm{p}<0.001)$. Both relationships indicate that animals with higher hindlimb range of motion also have more compliant muscles. See Fig. S1 in the supplementary online Appendix for information and sources on individual data points.

Fig. 2. Maximum in vivo knee range of motion and joint stiffness. (A) Xenopus laevis $(\mathrm{n}=3)$, (B) Kassina senegalensis $(\mathrm{n}=4),(\mathrm{C})$ Rhinella marina $(\mathrm{n}=3)$ and (D) Lithobates catesbeianus $(\mathrm{n}=3)$. Species photos are presented in order of increasing range of motion. Knee joint stiffness was characterized by measuring the passive force required to hold the knee statically at a given angle. Maximum range of motion during locomotion (any mode) is highlighted in grey for each species. Force was normalized by the square of the femur length to allow comparisons between animals that differ by an order of magnitude in body mass. Data for the right and left knee for each individual are shown; individuals are shown in different markers $(\square, \circ, \Delta, \diamond)$. Solid lines represent quadratic fit with the $95 \%$ confidence interval for the fit in dashed lines. The angle at which the quadratic fit was minimum differed significantly among species but did not correlate with range of motion. Kassina (B) and Lithobates (D) had a significantly higher quadratic coefficient, indicating stiffer knee joints. See Table 1 for quantification of stiffness parameters.

Fig. 3. Maximum in vivo ankle range of motion and joint stiffness. (A) Kassina senegalensis $(\mathrm{n}=4)$, (B) Rhinella marina ( $\mathrm{n}=4)$, (C) Lithobates catesbeianus $(\mathrm{n}=3)$ and (D) Xenopus laevis $(\mathrm{n}=3)$. Species photos are presented in order of increasing range of motion. Ankle joint stiffness was characterized by measuring the passive force required to hold the ankle statically at a given angle. Force was normalized by the square of the tibiofibula length to allow comparisons between animals that differ by an order of magnitude in body mass. Individuals are shown in different markers $(\square, \circ, \Delta, \diamond)$ and range of motion is highlighted in grey. Solid lines represent quadratic fit with the $95 \%$ confidence interval for the fit in dotted lines. The angle at which the quadratic fit was minimum differed significantly among species but did not correlate with range of motion. The quadratic coefficient was indistinguishable between Kassina (A) and Xenopus (D), but differed from that of the other two species. See Table 1 for quantification of stiffness parameters.

Fig. 4. Stiffness of muscle fiber bundles. (A) Rhinella marina, (B) Kassina senegalensis, (C) Lithobates catesbeianus, (D) Xenopus laevis. Different symbols represent different individuals, with muscle samples from both the right and left side. Solid lines represent lines of best fit for the pooled species data. Statistics were performed on the parameters of curves fit to individual data. The stiffness of the cruralis muscle was statistically indistinguishable among the four species, regardless of the measure of stiffness used. The Rhinella plantaris began developing tension at a significantly longer sarcomere length than the plantaris muscles of the other species, and both the Rhinella and Xenopus plantaris developed force at a lower initial rate during the exponential part of the curve. The open 
1 symbol individual in the Kassina plantaris plot (B) was an outlier and was not included in 2 statistical comparisons. See Table 2 for specific fit values.

Fig. 5. Normalized muscle moment arms (mean \pm sem). (A) Rhinella and Xenopus have relatively longer cruralis moment arms at the knee (normalized by femur length) than Kassina and Lithobates. (B) Kassina has a relatively shorter plantaris moment arm at the 7 ankle (normalized by tibiofibula length) than the other three species. See Section 3.4 for 8 mean femur and tibiofibula lengths for each species.

9 Fig. 6. Correlation between muscle stiffness metrics and joint range of motion for anurans. 10 Meta-data are presented in grey (see supplementary Fig. S1 and Table S1 for details) while 11 the new data from our study are shown in black (open symbols for cruralis, filled symbols 12 for plantaris). (A) L20, the length at which a muscle develops passive force equal to $20 \%$ of 13 its maximum isometric force (normalized to a sarcomere length of $2.2 \mu \mathrm{m}$ ), showed no 14 significant relationship with joint range of motion ( $\mathrm{F}=0.04, \mathrm{P}=0.84)$. Note the low $\mathrm{L} 20$ 15 values for Kassina and Xenopus. (B) Normalized muscle stiffness did not show a significant 16 relationship with joint range of motion $(\mathrm{F}=1.94, \mathrm{P}=0.20)$. Note the high normalized 17 stiffness of the Xenopus plantaris. 
Table 1. Knee and ankle joint stiffness, based on a quadratic fit to individual data. Curve minimum is the angle at which there is minimum resistance to bending and is significantly different among all species. Quadratic coefficient, k2, characterizes the width and slope of the curve. Knee: Kassina and Lithobates had a significantly higher k2 coefficient, indicating a narrower curve with higher slope. Ankle: Curve minimum was significantly different among all species. Kassina and Xenopus had significantly larger k2 coefficients than Lithobates and Rhinella.

\begin{tabular}{ccc}
$\begin{array}{c}\text { Range of } \\
\text { motion } \\
(0)\end{array}$ & $\begin{array}{c}\text { Joint angle at curve } \\
\text { minimum } \\
(\mathrm{o})\end{array}$ & $\begin{array}{c}\text { Quadratic coefficient, } \\
\text { k2 }\end{array}$ \\
\hline
\end{tabular}

\section{Knee}

Rhinella $(\mathrm{n}=6)$

Lithobates $(\mathrm{n}=4)$

Kassina $(\mathrm{n}=4)$

Xenopus $(\mathrm{n}=4)$

Ankle

Rhinella $(\mathrm{n}=5)$

Lithobates $(\mathrm{n}=5)$

Kassina $(\mathrm{n}=8)$

Xenopus $(\mathrm{n}=5)$
92.1

127.7

76.2

52

113

116.5

74.6

125.6
$82.9 \pm 0.16$

$116 \pm 0.34$

$88.0 \pm 0.46$

$65.4 \pm 5.12$

$82.0 \pm 0.42$

$84.3 \pm 0.17$

$106.9 \pm 0.43$

$119.5 \pm 5.05$

$$
\begin{gathered}
7.12 \times 10^{-5} \pm 5.6 \times 10^{-6} \\
3.58 \times 10^{-4} \pm 9.1 \times 10^{-5} * \\
2.00 \times 10^{-4} \pm 2.3 \times 10^{-5 *} \\
7.96 \times 10^{-5} \pm 4.4 \times 10^{-5}
\end{gathered}
$$

$9.82 \times 10^{-5} \pm 9.2 \times 10^{-6}$

$7.22 \times 10^{-5} \pm 3.03 \times 10^{-5}$

$2.7 \times 10^{-4} \pm 2.1 \times 10^{-5}$ *

$1.13 \times 10^{-4} \pm 1.8 \times 10^{-5}$ * 
Table 2. Fit parameters for cruralis and plantaris muscle fiber bundle stiffness. An exponential curve was fitted according to Brown et al. (1996b) to the stress-sarcomere length data for each species. The coefficients describe the slope of the curve at long sarcomere lengths (c), the curvature of the curve $(\mathrm{k})$ and the length at which tension begins to develop $\left(\mathrm{Lr}_{\mathrm{r}}\right)$. Best fit parameter \pm s.d. There was no significant effect of species on any of the fit parameters for the cruralis muscle. The plantaris muscle differed significantly between Rhinella and Xenopus (Tukey HSD, $\mathrm{p}=0.05$ ) in the k parameter. The $\mathrm{Lr}_{\mathrm{r}}$ for the Rhinella plantaris was significantly longer than those of the other species (Tukey HSD, $\mathrm{p}<$ 0.003 for all comparisons). The c parameter did not vary significantly.

\begin{tabular}{lccc} 
& $\mathbf{c}$ & $\mathbf{k}$ & $\mathbf{L}_{\mathbf{r}}$ \\
\cline { 2 - 4 } Cruralis & & & \\
Rhinella $(\mathrm{n}=4)$ & $0.35 \pm 0.32$ & $0.12 \pm 0.09$ & $2.68 \pm 0.40$ \\
Lithobates $(\mathrm{n}=10)$ & $2.72 \pm 4.88$ & $0.58 \pm 0.87$ & $3.60 \pm 1.04$ \\
Kassina $(\mathrm{n}=6)$ & $21.62 \pm 37.98$ & $0.09 \pm 0.05$ & $2.90 \pm 0.46$ \\
Xenopus $(\mathrm{n}=5)$ & $2.04 \pm 1.91$ & $0.19 \pm 0.12$ & $3.33 \pm 0.58$
\end{tabular}

\section{Plantaris}

$\begin{array}{lcll}\text { Rhinella }(\mathrm{n}=7) & 12.65 \pm 27.59 & 0.33 \pm 0.18^{*} & 3.99 \pm 0.59^{*} \\ \text { Lithobates }(\mathrm{n}=7) & 1.27 \pm 2.51 & 0.29 \pm 0.23 & 2.93 \pm 075 \\ \text { Kassina }(\mathrm{n}=5) & 8.55 \pm 8.94 & 0.13 \pm 0.03 & 2.74 \pm 0.16 \\ \text { Xenopus }(\mathrm{n}=5) & 3.10 \pm 4.26 & 0.07 \pm 0.04^{*} & 2.41 \pm 0.10\end{array}$


Table 3. Body and muscle mass for four species of frogs. For body mass data, Rhinella $\mathrm{n}=3$ animals, Lithobates $\mathrm{n}=4$, Kassina $\mathrm{n}=6$, Xenopus $\mathrm{n}=3$; for cross-sectional area (csa) data, Rhinella $\mathrm{n}=6$ animals, Lithobates $\mathrm{n}=11$, Kassina $\mathrm{n}=4$, Xenopus $\mathrm{n}=4$. Data also from Chadwell et al. (2012) for Rhinella and Lithobates, Richards and Biewener (2007) for Xenopus.

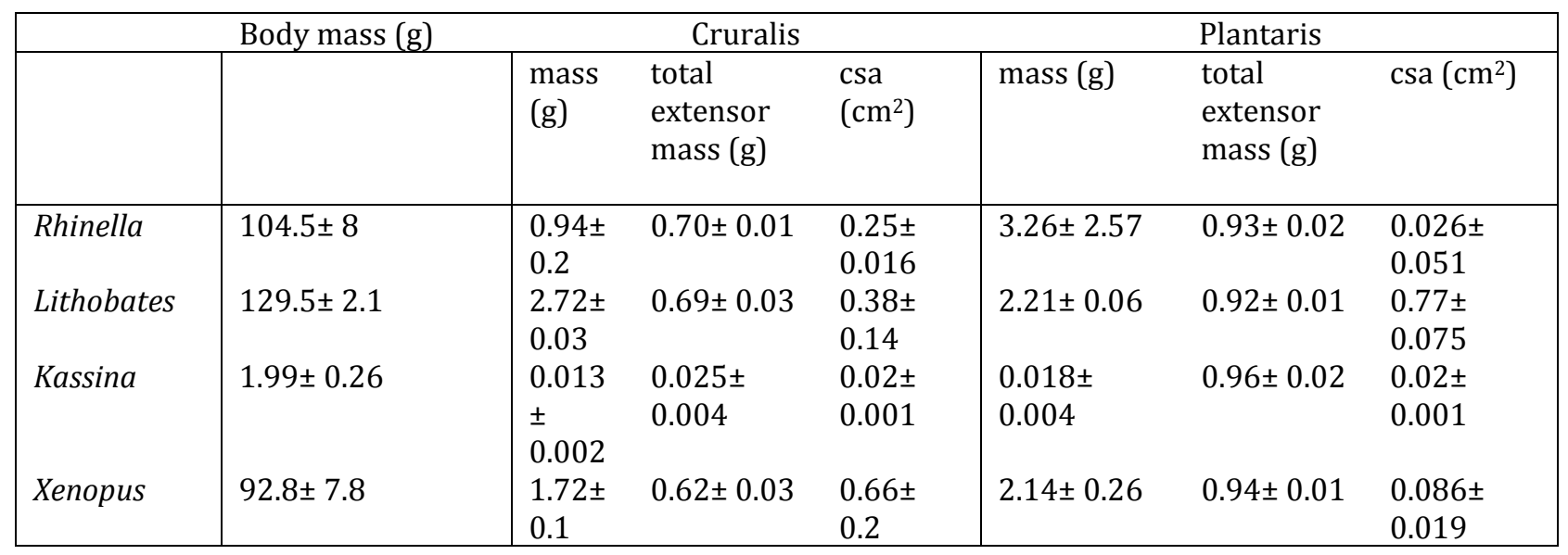




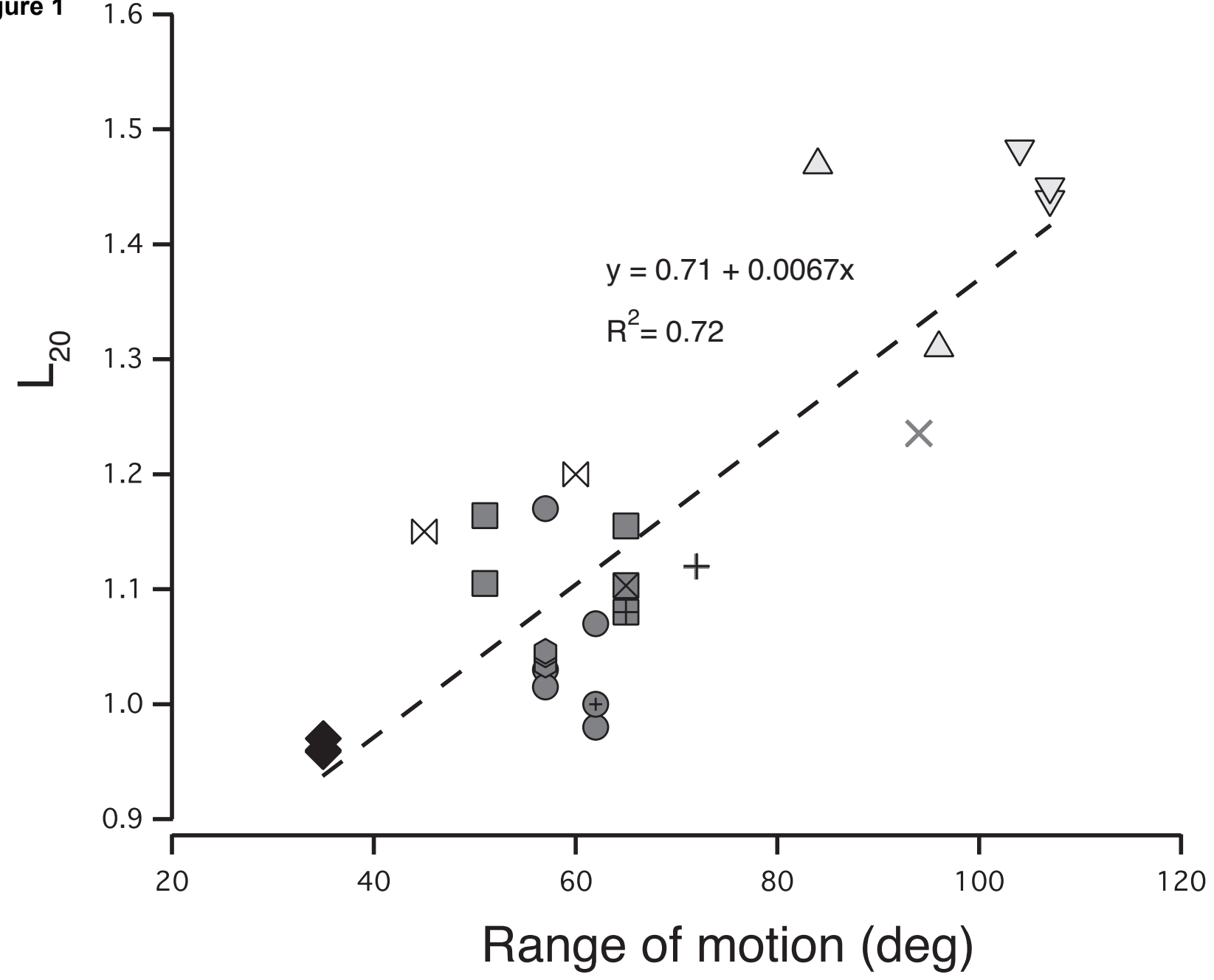

B.

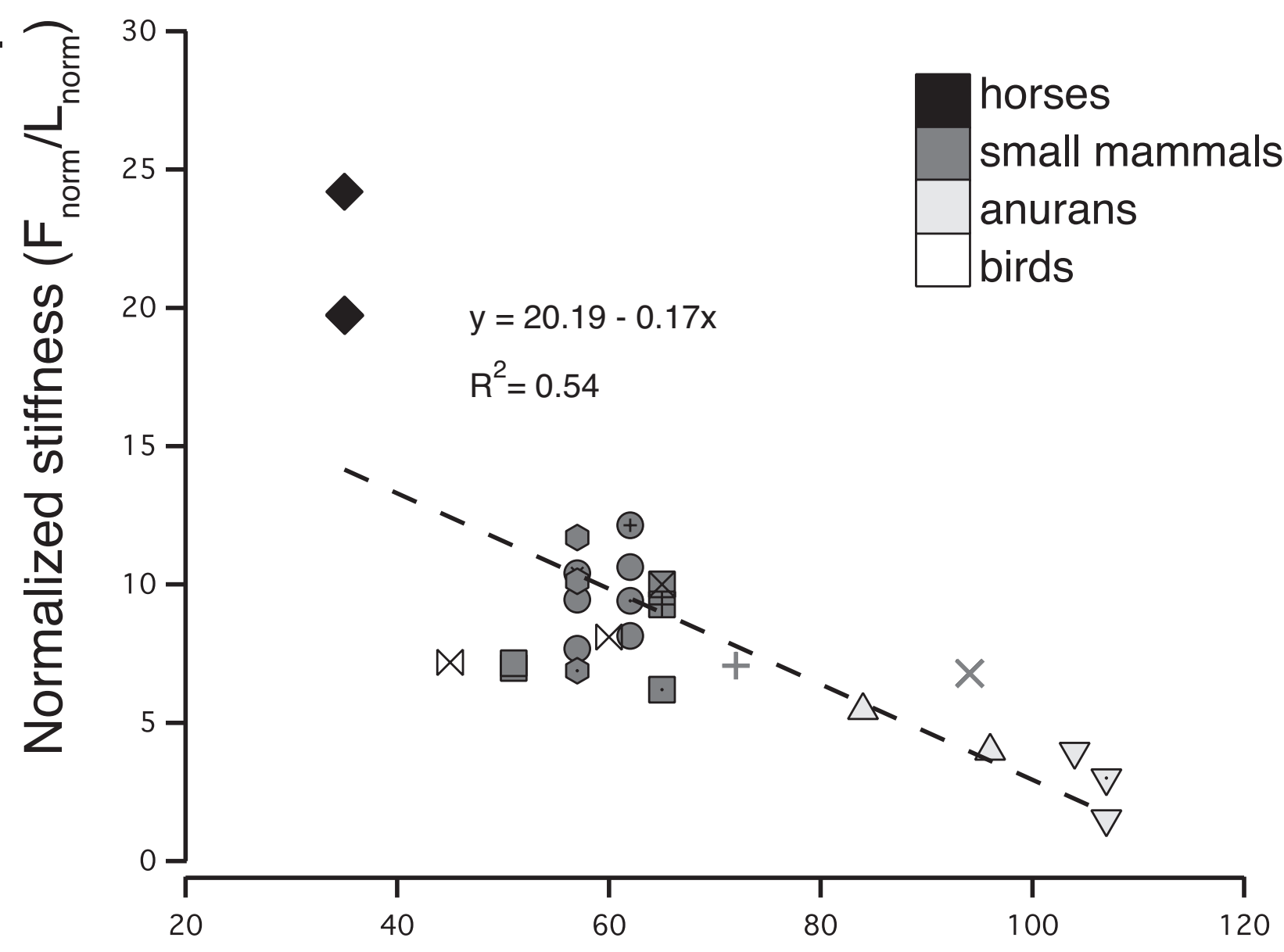

Range of motion (deg) 
Figure 2

range of motion
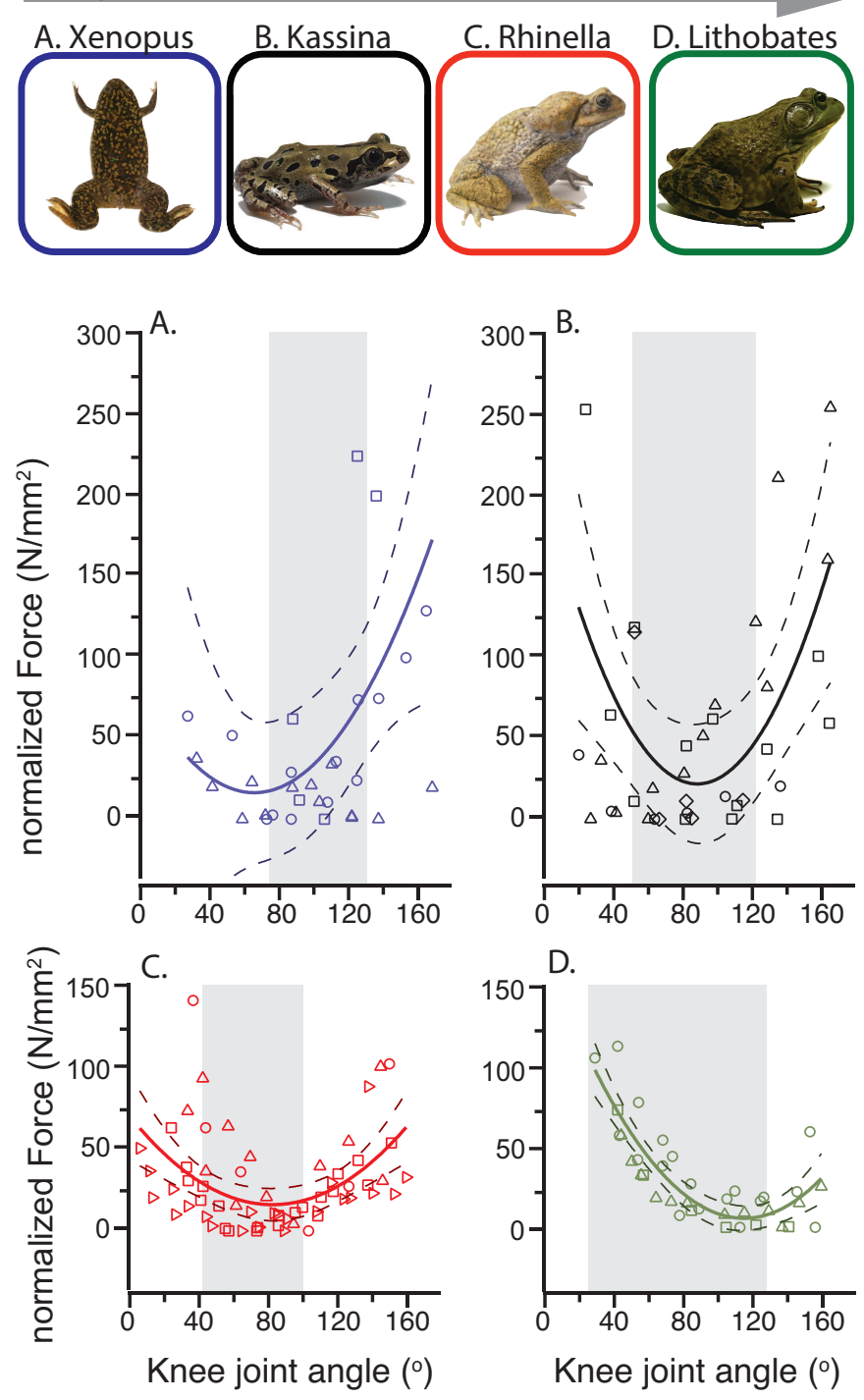
Figure 3

range of motion
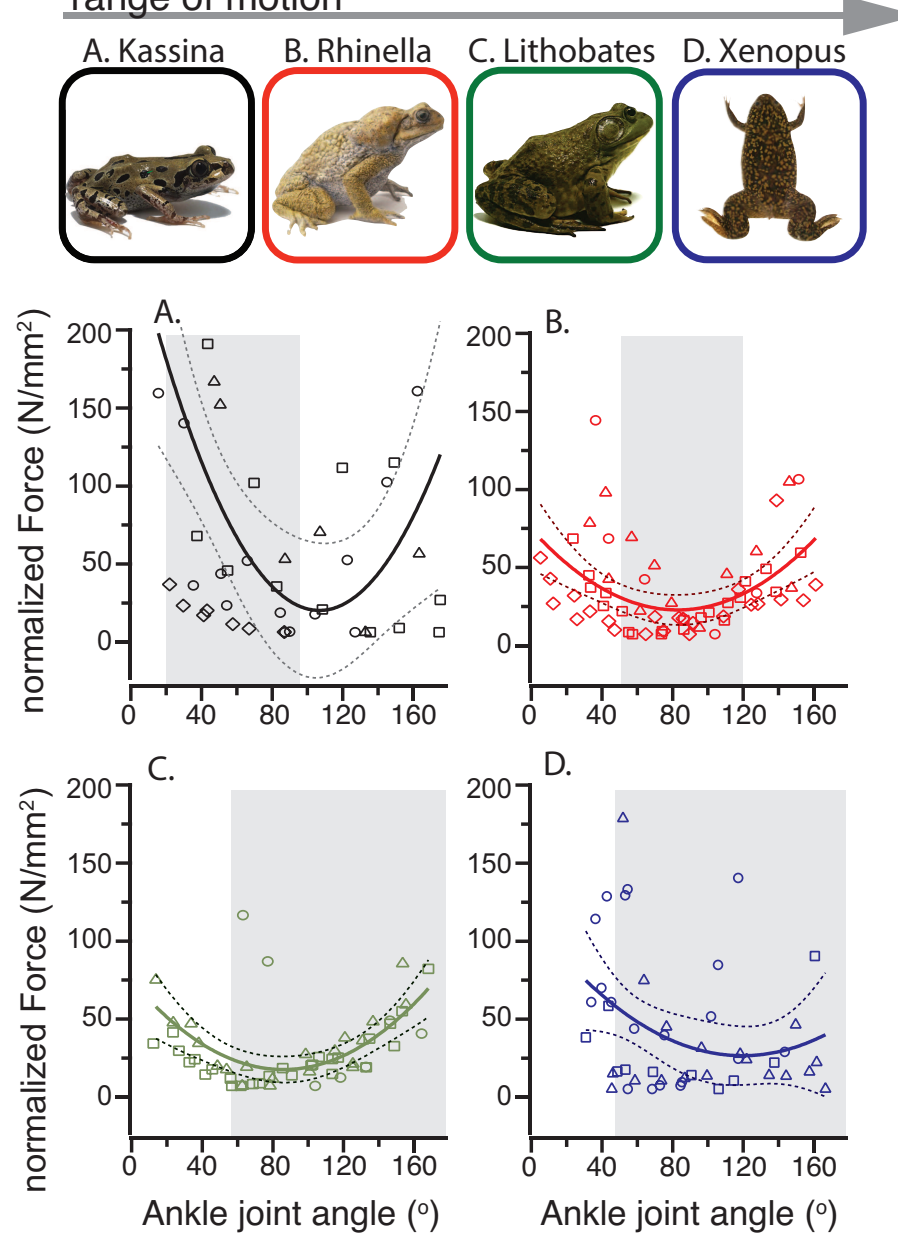


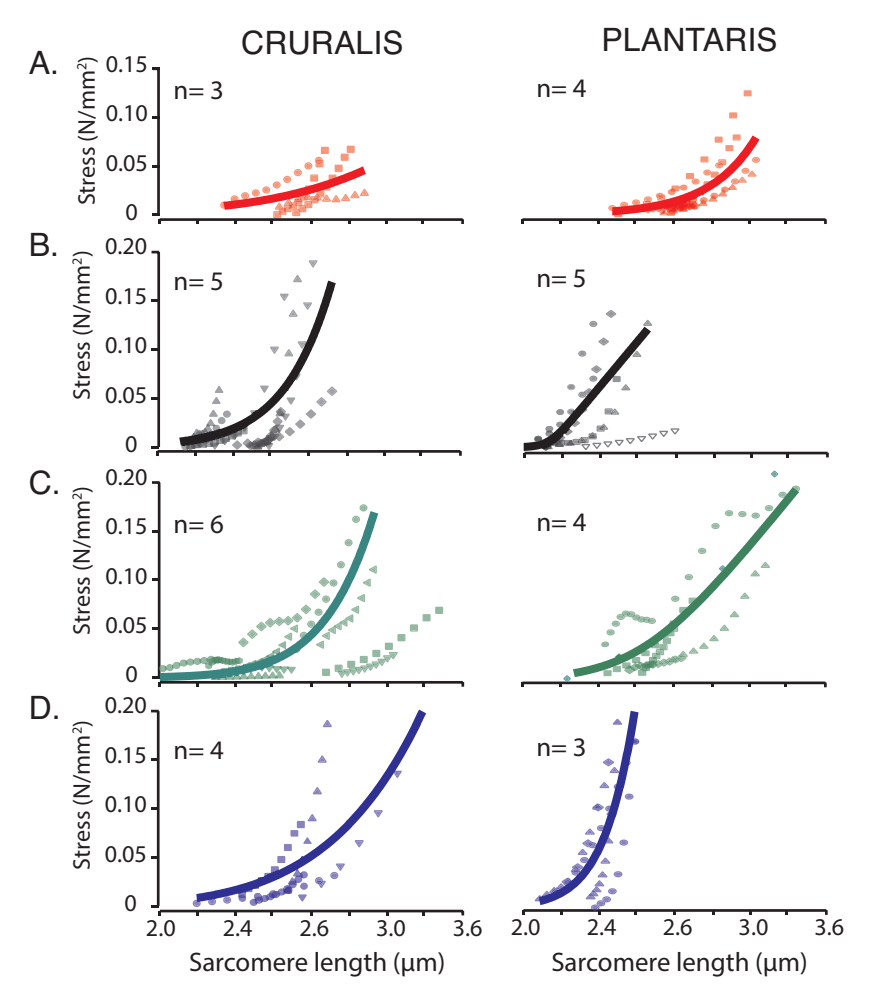

$\mathrm{n}=5$

Figure 4

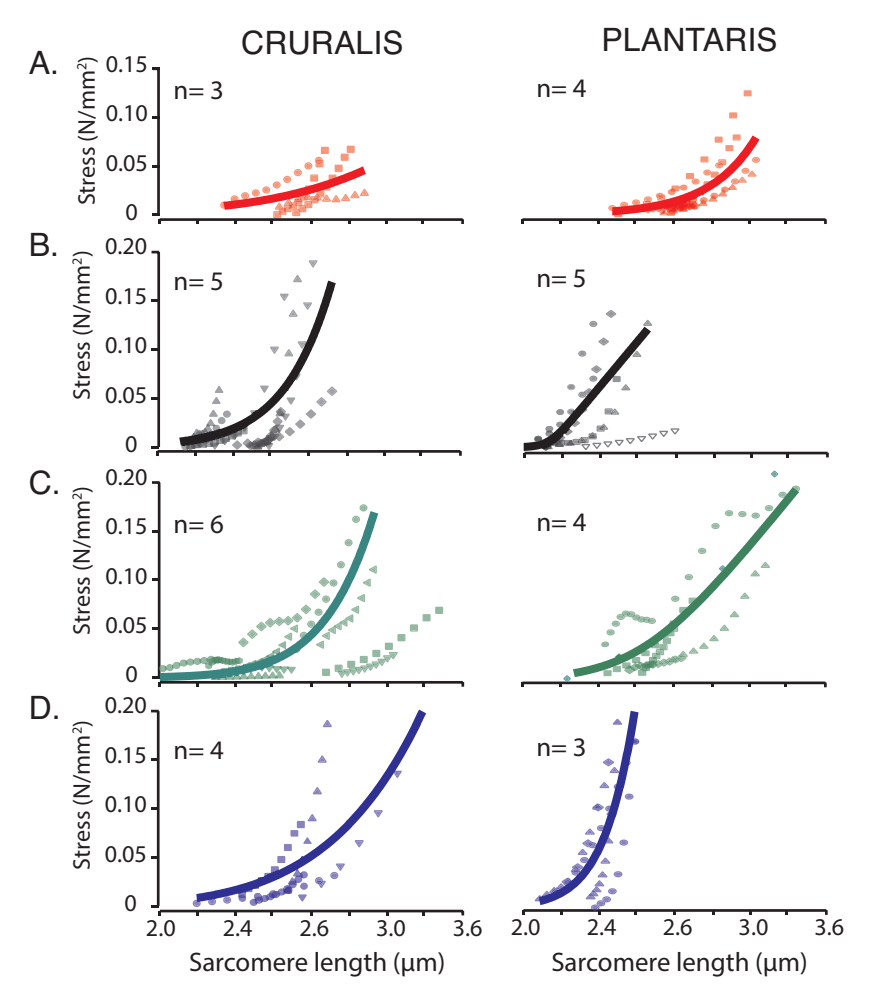


A. CRURALIS

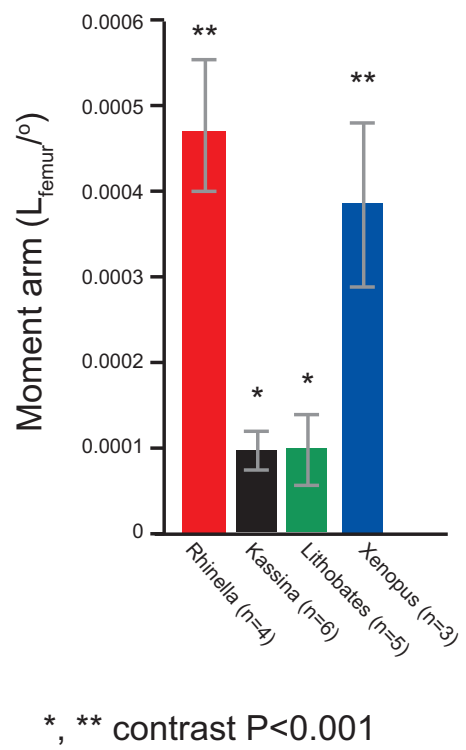

${ }^{*},{ }^{* *}$ contrast $\mathrm{P}<0.001$
B. PLANTARIS

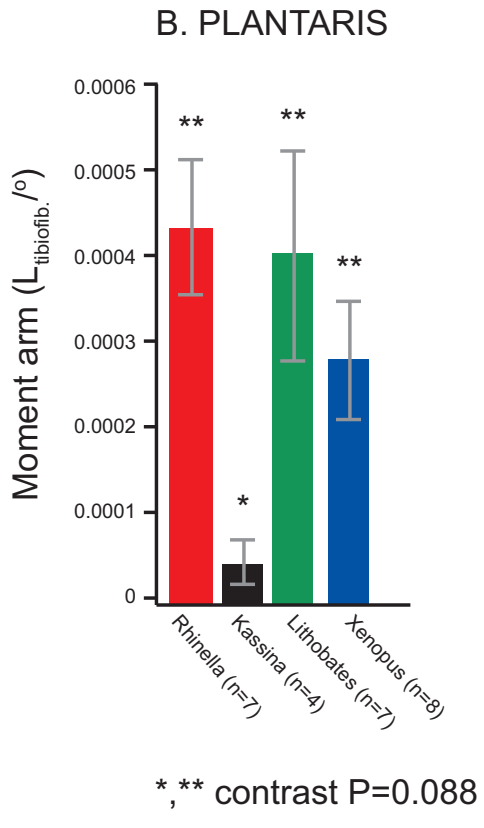

contrast $\mathrm{P}=0.088$ 
